TEMA DE ACTUALIDAD

\title{
Políticas de ingreso a las universidades
}

\section{Entry policies in universities}

\author{
* Mora Rojas, C.O. \\ ${ }^{1}$ Universidad Nacional de Asunción (UNA). Facultad de Ciencias Químicas. \\ Paraguay
}

\section{RESUMEN}

En este trabajo se presentan los resultados de investigación sobre las políticas de ingreso a las universidades de América Latina y el Caribe, comparadas a las políticas de Paraguay, considerando que cada sociedad tiene su realidad social, económica, cultural y política, reflejada en sus políticas públicas. El presente trabajo aborda el tema de las políticas de ingreso o acceso a las universidades de América Latina y el Caribe, considerando a la universidad como institución dinámica y con autonomía. La metodología utilizada fue análisis documental, mediante revisión bibliográfica, con el objetivo de describir las políticas de ingreso a las universidades de la región estudiada.

Palabras clave: universidades, políticas de ingreso, América Latina y el Caribe, Paraguay.

\section{ABSTRACT}

This paper presents the results of research on the policies of admission to the universities of Latin America and the Caribbean, compared to the politics of Paraguay, considering that each society has its social, economic, cultural and political reality reflected in his policies. This paper addresses the issue of policies of income or access to the universities of Latin America and the Caribbean, whereas to the University as a dynamic institution with autonomy. The methodology used was documentary analysis, through review of the literature, aiming to describe the policies of admission to the universities of the region studied.

Keywords: universities, political entry, Latin America and the Caribbean, Paraguay.

\section{INTRODUCCIÓN}

El número de universidades en la región Latinoamericana y especialmente en Paraguay ha crecido casi exponencialmente en los últimos treinta años, en cuanto al aumento de las mismas, y con este crecimiento se establecen las exigencias de calidad, tanto por las agencias de acreditación como por la propia sociedad. Este crecimiento permitió entre otras características la Existencia de diversidad de tipologías de estas instituciones de educación superior. El presente artículo de actualidad persigue describir las políticas de ingreso a las universidades de América Latina y el Caribe, mediante la compilación, revisión bibliográfica y análisis de información con rigor científico. 


\section{POLÍTICAS DE INGRESO A LAS UNIVERSIDADES DE AMÉRICA LATINA Y EL CARIBE}

La universidad actual tiene el desafío de insertarse en un mundo complejo, con nuevos paradigmas de profesionalismo y competencias emergentes asociadas a la sociedad del conocimiento que se hallan en constante aumento y transformación. (González \& Espinoza, 2008)

La universidad que conocemos hoy en día es grande, compleja, plural, con una organización un tanto diversa, aunque con características propias, con principios que constituyen el concepto de universidad, siendo algunos principios de carácter permanentes tales como valores, principios de los miembros y las virtudes de los estudios. Es una institución dinámica y a la luz de esos principios se debe reflexionar sobre la educación superior y su impacto en la sociedad moderna. (González, 1997)

Uno de esos principios es la autonomía universitaria, que permite a las universidades entre otras facultades determinar el sistema de ingreso o acceso a la misma. Esto ha llevado a una gama de mecanismos de ingreso a las universidades, cada una de ellas según sus principios misionales propios. Estos variados sistemas de ingreso son lo que se presenta en la siguiente investigación tanto a nivel de América Latina y el Caribe.

En América Latina y el Caribe, existen modalidades de ingreso a la Educación Superior, que pueden ser denominadas de "selección explícita" y de "selección implícita", la primera hace referencia a la modalidad en que los criterios de selección, están definidos a nivel nacional, o a nivel de cada institución; y la segunda cuando se refiere al caso del ingreso irrestricto. Cada variante tiene sus aspectos negativos y positivos sin que ninguna de ellas haya logrado conseguir por lo menos equilibrar el binomio "equidad-calidad" (García Guadilla, 1996).

En la década del 90 , se establecieron amplios debates en los que los organismos internacionales, por un lado, daban una mirada a la educación superior como agente para el desarrollo económico y social, con una visión más economicista en el concierto de la educación superior, el rol del Estado y la autonomía institucional, y por otro lado una visión más humanista de la educación superior, que es planteada por otros sectores. De este amplio debate surge que las Constituciones de América Latina hacen responsable al Estado de garantizar el acceso y la calidad de la educación como derecho consagrado (Robledo, 2017).

El ingreso, retención y egreso en las universidades forman parte de un incesante derrotero, con características propias y que impactan sobre el posible éxito o fracaso del alumno. Difícilmente un sistema universitario será exitoso, si el sistema de ingreso es poco selectivo. Se debe tener en cuenta que el acceso, ingreso y egreso universitario representan tres instancias diferenciadas, flojamente asociadas, que toman lugar en dispares momentos del tiempo. Habitualmente se consideran como sinónimos el ingreso y el acceso a la universidad; sin embargo, el acceso es un concepto más amplio pues conlleva a una serie de factores relacionados con el perfil socioeconómico del candidato a estudios universitarios. El ingreso irrestricto que caracteriza a la educación superior argentina puede llevar a varias consecuencias; como ser que los jóvenes que acceden por este medio a la educación superior universitaria no estén preparados para enfrentar los retos que implica este nivel de formación, puesto que es menos selectivo (Rabossi, 2014). 
La preocupación por las pruebas de ingreso a la universidad es una tendencia mundial creciente y sus efectos se analizan con base a estudios de seguimiento que permiten discriminar respecto a la mejor capacidad predictiva del desempeño académico estudiantil en la universidad. En Chile, durante varias décadas se aplicó la Prueba de Aptitud Académica (PAA) como sistema de ingreso a las Universidades, en conjunto con las notas de enseñanza media (NEM), así se otorgaba un puntaje de ingreso a la educación universitaria para cada estudiante. Posteriormente llegó a aplicarse la Prueba de Selección Universitaria (PSU), que evalúa habilidades intelectuales, modos de operación y métodos generales aplicados a la resolución de problemas en las pruebas de lenguaje y comunicación, matemática, historia y ciencias sociales, y ciencias, que incluye biología, física y química (Vergara Díaz \& Peredo López, 2017).

En Ecuador fueron realizadas reformas a la educación superior en los años 2008 y el 2012, de modo a eliminar las barreras de acceso a la universidad, mediante la aplicación de la política de gratuidad de la educación superior aprobada en la Constitución del 2008; y, por otro lado, implementar un examen de ingreso a las universidades, así como el cierre de universidades de deficiente calidad en el año 2012. Estas reformas han presentado resultados positivos en cuanto permitieron la elevación del número de la matrícula debido a la gratuidad; mientras que el examen de ingreso y el cierre de las universidades de mala calidad tuvieron efectos negativos en la matrícula. Además, se han hallado mejoras en términos de equidad, incrementando el acceso al sistema de los estratos sociales medios (Ponce \& Carrasco, 2016).

En diferentes países y aún al interior de un mismo país la llegada a la educación superior de carácter universal presenta innumerables diferencias, tornándose incluso en una arista de inequidad, que más aún puede aumentar la brecha de desigualdades y oportunidades de acceso ya vigentes. El escaso acceso a la ciencia y tecnología de ciertos grupos excluidos, en general de bajos ingresos, perjudica finalmente a la sociedad toda; siendo ésta la razón fundamental y para que las políticas públicas vinculadas al tema del acceso sean equitativas e inclusivas (Pesántez Avilés, Martín, \& Bojorque, 2015).

Diversas universidades argentinas suscriben políticas de ingreso coherentes en cuanto a la inclusión plena de los ingresantes a la vida universitaria. La población estudiantil de la universidad argentina se caracteriza por la diversidad social, cultural y por trayectorias educativas disímiles y desiguales, situación que se reconoce como el producto de un sistema de enseñanza fragmentado y segmentado (Garratte, Montenegro, Fava, \& Alcoba, 2018).

En cuanto a Costa Rica no existen exámenes nacionales de ingreso a la educación superior, ni políticas públicas en las que se establezcan los criterios de ingreso a las universidades públicas o privadas; sólo existen requisitos de ingreso a este nivel educativo: el de disponer del título de educación secundaria $y$, en algunas de las instituciones universitarias se administra un examen de admisión, con políticas o criterios de admisión determinados por cada una de las instituciones universitarias (Centro Interuniversitario de Desarrollo - CINDA, 2016).

Finalmente se observa que resulta difícil para los responsables de establecer las políticas de ingreso a la universidad plantear solución al dilema equidadcalidad en el acceso, ya que el mismo está muy asociado a problemas que podrían ser denominados determinantes sociales que son debidos a múltiples variables y factores, y en su mayoría son externos a la educación superior, como lo son las grandes desigualdades sociales, económicas, culturales y 
escolares, que caracterizan a la mayoría de nuestros países, y a las formas de articulación exclusivamente vertical entre los distintos niveles de educación y propias de los actuales sistemas educativos (García Guadilla, 1996) y si se asume que el sistema educativo actual falló porque mató la creatividad, se debe tener mucha creatividad para equilibrar las fuerzas de la equidad y calidad (Díaz Sánchez-Aguilera \& Alonso Morante, 2017).

\section{POLÍTICAS DE INGRESO EN LAS UNIVERSIDADES PARAGUAYAS}

En la postguerra contra la Triple Alianza, en el año 1887 se creó el Consejo Superior de Educación y se dictó una Ley de Enseñanza Obligatoria. La Universidad Nacional de Asunción (UNA) fue fundada en base a dicha ley, en fecha 24 de setiembre de 1889, con las Facultades de Derecho y Ciencias Sociales, de Ciencias Médicas y de Matemáticas (Benítez, 1981).

Mediante Decreto del Poder Ejecutivo promulgado el 22 de enero de 1960 es autorizado el funcionamiento y el 13 de febrero del mismo año, la Conferencia Episcopal Paraguaya (CEP) dispuso la creación de la Universidad Católica "Nuestra Señora de la Asunción" (UCA) mediante el funcionamiento del Instituto Superior de Teología, marcando con esto una evolución de la educación superior universitaria. Esta creación fue histórica, pues con ella desaparece -en parte por lo menos- el monopolio de la UNA sobre la educación universitaria paraguaya (Rivarola, 2003).

Se ha visto una expansión de las universidades privadas en la década de los 90 y en los comienzos del siglo XXI, a raíz de las demandas sociales de la época, con un crecimiento vertiginoso. Las sustentaciones legales de las creaciones de las mencionadas instituciones universitarias han sido las leyes según el momento histórico de su creación.

Según las páginas oficiales de la Agencia Nacional de Evaluación y Acreditación de la Educación Superior (ANEAES), y del Consejo Nacional de Educación Superior (CONES)

(CONES, 2018), el Paraguay cuenta con universidades, de gestión oficial y privada.

Tabla 1: Frecuencias de universidades, según tipo de gestión.

\begin{tabular}{lll}
\hline Tipo de Gestión & Frecuencia absoluta & Frecuencia relativa \\
\hline Oficial & 8 & $15 \%$ \\
Privada & 47 & $85 \%$ \\
\hline
\end{tabular}

Las universidades que fueron creadas por decreto del poder ejecutivo se realizaron en el marco de la Ley 828/80 (ANEAES, 2018).

En la Tabla 1 está considerada, la Universidad Politécnica Taiwán Paraguay, que es creada en el marco de la conmemoración de los 60 años de relaciones diplomáticas entre Taiwán y Paraguay, idea materializada el 5 de Diciembre del 2017 con la firma de "La Carta de Intención" entre el Embajador de la República China (Taiwán) en el Paraguay, Alexander Yui, y el Ministro de Industria y Comercio, Gustavo Leite, promulgándose el día 31 de mayo del 2018 la Ley No 6.096/18 "que crea la Universidad Politécnica Taiwán- Paraguay" (MIy C, 2014).

El Paraguay experimenta una época de oportunidades de desarrollo, que abarca también a todo el subcontinente de América Latina y el Caribe. Para el aprovechamiento de esas oportunidades, una de las premisas es el 
fortalecimiento de la educación universitaria. La oferta universitaria en el país está descentralizada e impulsada por varias lógicas, que pueden develarse analizando su desarrollo, una de ellas es la lógica de mercado, en donde prima la necesidad del estudiante, que es la de contar con una Universidad cercana a su residencia de modo a ahorrar costos y esfuerzo. También se da una lógica sociopolítica, pues las regiones y sus ciudades al disponer de Universidades en la propia ciudad ganan signos de prestigio, identidad y de autonomía (Viceministerio de Educación Superior, 2013).

Como describe Parra (2012), las tres formas básicas de acceder a las Universidades paraguayas son:

a) La realización de un curso probatorio de ingreso de carácter eliminatorio, donde los estudiantes que logran los mejores puntajes acceden a la universidad y aquellos que logran los puntajes más bajos quedan afuera.

b) La realización de un curso de admisión de carácter no selectivo, que generalmente tiene como objetivo dotar de conocimientos o competencias generales a los futuros universitarios. Esta modalidad a diferencia de la anteriormente citada no es de carácter eliminatorio.

c) El acceso directo, en el cual el estudiante se inscribe directamente en la carrera que va a cursar.

Se debe agregar a las tres formas básicas de acceso, el tradicional examen de ingreso sin curso alguno.

El sistema de acceso o ingreso a las universidades debe ser analizado en sus dos aristas: equidad y calidad. Equidad en cuanto a oportunidades a la educación superior como un bien público y humano. Por otra parte la calidad en cuanto a excelencia académica, y en estas aristas deben conciliarse los recursos disponibles en el sistema (González \& Espinoza, 2008).

Las distintas universidades deben presentar consistencia interna y en el marco de dicha consistencia deben ser evaluados sus procesos, incluyendo el de acceso (De Vincenzi, 2016).

La educación es la principal fuerza creadora de las potencialidades humanas, los cuestionamientos del para qué se educa hoy, y el cómo se educa hoy, en los tiempos actuales considerados como la era de mutaciones ecológicas y tecnológicas, debe llevar al repensamiento del sistema educativo en su contexto global, incluyendo los sistemas de acceso a las universidades (Pérez Lindo, 2009).

Al realizar el análisis del ingreso en las universidades paraguayas se procede al análisis basado en el tipo de gestión universitaria.

Las universidades del sector oficial prácticamente presentan el denominador común de un sistema de admisión, cuyo objetivo general es la selección de los mejores postulantes, enmarcados por la dimensión de los recursos, que determina en las unidades académicas y en cada una de las carreras de grado, el número de plazas disponibles. Este punto constituye el centro de gravedad, el punto de apoyo o de equilibrio entre la equidad y la calidad. Se observa, que aunque exista el denominador común, también la observancia de mecanismos o escenarios disímiles en el proceso de admisión.

La oferta académica es muy amplia, por tanto se realizan generalizaciones, con algunos casos particulares a modo de ejemplificar cada situación.

El estatuto general de la UNA, recientemente aprobado y puesto en vigencia, reza en el artículo 144

Para ingresar a la UNA se requiere: 
a) Haber egresado de la educación media con título de bachiller o equivalente. El título y el certificado de estudios de bachiller o equivalente deberán estar registrados y legalizados en el Ministerio de Educación y Ciencias (MEC) y en la UNA, conforme a las disposiciones legales vigentes.

b) Cumplir los demás requisitos establecidos en los reglamentos de la UNA y de sus respectivas unidades académicas (UNA, 2018).

Actualmente la UNA posee catorce unidades académicas, con las recientes transiciones del Instituto "Dr. Andrés Barbero" a Facultad de Enfermería y Obstetricia "Dr. Andrés Barbero" y de la Escuela de Ciencias Políticas a Facultad de Ciencias Políticas. Cada una de estas catorce unidades académicas posee su sistema de admisión, siempre en el marco de lo que establece el Estatuto de la UNA. Así se distinguen en sus distintas unidades académicas:

- Admisión mediante exámenes de Ingreso, con preparación autónoma de los postulantes.

- Admisión mediante exámenes de Ingreso, con preparación de los postulantes mediante cursillos o tutoriales, ofertados por la institución, sin ser éstos de realización obligatoria.

- Admisión mediante exámenes de Ingreso, con preparación de los postulantes mediante cursillos ofertados por la institución, de realización obligatoria.

- Cursos Probatorios de Ingresos y Cursos Propedéuticos.

Se podría entonces generalizar que en la UNA y también en las otras universidades nacionales, que son de gestión oficial, el mecanismo de selección de ingresantes, es explícito (García Guadilla, 1996).

En cuanto a las universidades de gestión privada, en la Universidad Católica el postulante debe realizar un curso de admisión, que como componente del sistema de admisión, mediante un proceso de integración de saberes y experiencias del alumno permite promover y verificar las competencias básicas para la vida universitaria (Parra, 2012).

En cuanto a la admisión a la Universidad del Norte:

Son condiciones para solicitar la admisión de un alumno a la Universidad:

Para estudiantes paraguayos:

Haber concluido, aprobado y obtenido el título de Bachiller Nacional o el equivalente en el extranjero.

Presentar el título de Bachiller Nacional o equivalente en el extranjero; original del certificado de estudios, ambos visados y legalizados por el Ministerio de Educación y Ciencias de la República del Paraguay.

Presentar copia de la cédula de Identidad.

Para estudiantes extranjeros:

Llenar correctamente la solicitud de ingreso, que será dirigida a la Junta de Admisión de la Universidad del Norte. La Junta de Admisión, está integrada por: los Miembros del Consejo Superior Universitario, el Decano de la Facultad a la que aplica, el Director de Carrera en el caso que corresponda. La decisión de la Junta de Admisión es inapelable.

Presentar el título equivalente al de Bachiller Nacional y certificado de estudios, en dos copias, debidamente autenticadas por el Ministerio de Educación del país de origen o equivalente, por el Ministerio de Relaciones Exteriores del país de origen y del nacional, y por el Ministerio de Educación y Ciencias de la República del Paraguay.

Las personas que deseen inscribirse y no provengan de un país hispano parlante, deberán tomar un examen ante la Junta de Admisión con el fin de demostrar suficiente dominio del idioma castellano (hablar, leer y escribir). 
Presentar copia autenticada del País de Origen con la visa de Estudiante otorgada por la República del Paraguay (UniNorte, 2018).

Otra forma de acceso a las universidades paraguayas de gestión privada, es la ofertada por la Universidad del Pacífico, mediante un Curso Propedéutico de la carrera de Medicina, dirigido a postulantes al ingreso a dicha carrera, que cuenten con bachillerato concluido o cursando el último año de la Educación Media (Universidad del Pacífico, 2018).

En general en las universidades de gestión privada del Paraguay, predomina el mecanismo de selección de ingresantes implícito (García Guadilla, 1996).

\section{DISCUSIÓN}

Cada universidad aplicando su sistema de admisión, por lo que se puede apreciar, busca el justo medio entre equidad y calidad.

La diversidad de mecanismos de admisión a las universidades de toda América Latina y el Caribe, es debido fundamentalmente a cuestiones o coyunturas políticas y sociales.

En el Paraguay las diversas modalidades de admisión obedecen a las políticas y realidades de cada universidad en particular. En las universidades de gestión pública el factor predominante es el de los recursos financieros, lo que debe obligar a estas instituciones a realizar mayores gestiones ante las instancias gubernamentales correspondientes para el logro de dichos recursos, además se suma la necesidad de una mayor visibilidad del impacto de la labor académica en la sociedad; de modo que la sociedad toda, apoye los reclamos de aumento presupuestario destinado a la educación en todos sus niveles.

En cuanto a las universidades de gestión privada que cuentan con requerimientos mínimos de admisión, lleva a la reflexión de la necesidad del establecimiento de perfiles nacionales de admisión para las diversas ofertas académicas y que éstos sean incorporados y evaluados en los procesos de acreditación de carreras; aunque actualmente se presentan los perfiles de admisión en los informes de autoevaluación, no existen perfiles mínimos. Esto debería aplicarse para las carreras tanto de universidades de gestión privada como pública, teniendo en cuenta que el Estado es el responsable de velar por la calidad de la Educación Superior.

Es pertinente incorporar la aplicación de test psicotécnicos a los postulantes antes de los exámenes de admisión o inscripción directa a las carreras, a los efectos de realizar una orientación a los interesados respecto a sus cualidades vocacionales, teniendo en cuenta la existencia de jóvenes que seleccionan carreras equivocadas y que luego terminan desertando tanto en instituciones públicas como en las privadas, ocasionando perjuicios económicos y de tiempo a sí mismos y también a terceros, al dejar vacante la plaza por el abandono. El momento oportuno para la realización de los test es en el último curso de la Educación Media. Se ve la necesidad de fortalecer la aplicación de estos test en todos los colegios del país, independientemente si son de gestión pública o privada, lo que a su vez implica la incorporación de psicólogos en todas las instituciones de Educación Media. Se constata la existencia de alumnos que han sido educados con adecuaciones curriculares en la media, que llegan a la Universidad y ésta ya no cuenta con ese sistema. Podría ser una buena opción de que la Universidad tenga un sistema de nivelación, de forma a disminuir la brecha de inequidad por lo anteriormente mencionado.

En cuanto a equidad, en el Paraguay los postulantes pueden acceder a subvención o becas dadas por las Municipalidades y Gobernaciones entre otras; 
como premio a la excelencia al desempeño del postulante en la Educación Media.

Un aspecto interesante que podría contribuir a la excelencia académica de los postulantes es la necesidad de una ponderación del promedio de la Educación Media, tanto para el ingreso a nivel de las universidades de gestión pública o privada, con un incentivo, que podría ser establecido por cada universidad. Sería interesante que el sistema educativo de la Media cuente con un sistema de evaluación global, un único instrumento para todos los colegios.

Otra dimensión para la mejora de la inserción de los postulantes a los estudios universitarios, es el fortalecimiento de la articulación entre la Educación Universitaria y la Educación Media. Esta relación ya está iniciada con el "Programa de Capacitación de los Educadores para el Mejoramiento de los Aprendizajes de niños, niñas, jóvenes y adultos del Paraguay" (Procema), donde profesores universitarios realizan las capacitaciones a los docentes del MEC, muchos de los cuales no cuentan con la Licenciatura de la disciplina enseñada (Ministerio de Educación y Ciencias, Paraguay, 2017).

Así mismo la importancia de potenciar la Extensión Universitaria, en lo que se refiere a la capacitación de los docentes del MEC, incluyendo hasta el nivel de la Escolar Básica. La Universidad contribuiría grandemente a la educación aumentando los espacios de interacción con la Educación Media, con actividades de Extensión tales como Tutorías a Docentes del MEC y apertura de sus laboratorios para la ejecución de proyectos y experimentos. De esa forma la Universidad estaría cumpliendo un fuerte rol social en cuanto a la creación de un Semillero de Investigadores, tanto en estamento estudiantil como en el docente y en la mejora de la educación paraguaya.

\section{CONCLUSIÓNES}

Con el análisis se concluye:

- En toda América Latina y el Caribe se presentan variados mecanismos de ingreso a las universidades.

-El Paraguay presenta mecanismos de selección endógena o dentro del sistema universitario y selección implícita o ingreso irrestricto; los primeros predominan en las universidades de gestión pública y los últimos en las de gestión privada.

- El sistema de admisión en varias unidades académicas tanto de gestión pública o privada, ha ido evolucionando en la región latinoamericana.

- Las políticas de ingreso no siempre resultan en un verdadero acceso a la educación superior, a la permanencia y al egreso de los estudiantes.

- Aspecto común en las políticas de ingreso de América Latina y el Caribe es la búsqueda de equilibrio entre calidad y equidad.

\section{REFERENCIAS BIBLIOGRÁFICAS}

ANEAES (2018). Recuperado De Página oficial de Agencia Nacional de Evaluación y Acreditación de la Educación Superior: Nómina de universidades del sector oficial y privado con marco legal de aprobación para su funcionamiento: http://www.aneaes.gov.py/aneaes/index.php/ct-menu-item-31

Centro Interuniversitario de Desarrollo CINDA. (2016). Educación Superior en Iberoamérica. Informe 2016. Santiago-Chile: RIL R Editores.

CONES (2018). Página oficial del Consejo Nacional de Educación Superior: Registro Nacional de Ofertas Académicas. Recuperado de: http://www.cones.gov.py/carreras-y-programas-habilitados-por-el-cones/

De Vincenzi, R. (2016). Aseguramiento de la calidad: entre la autonomía institucional y la intervención estatal. Buenos Aires: Prometeo Libros. 
Díaz Sánchez-Aguilera, E., \& Alonso Morante, A. (2017). El sistema educativo actual falló porque mató la creatividad. World Economic Forum.

Facultad de Ciencias Químicas. Universidad Nacional de Asunción. (2018). Recuperado de http://www.qui.una.py/

Facultad de Ciencias Químicas. UNA. (2015). Curso probatorio de ingreso para la carrera de Nutrición 2015-2016. San Lorenzo, Paraguay. Instructivo impreso

Facultad de Ciencias Químicas. UNA. (2016). Instructivo CPI 2016. Recuperado de www.qui.una.py/files/cpi/Instructivo\%20CPI\%202016.pdf. de http://www.qui.una.py/

Facultad de Ciencias Químicas UNA (2019) Guía del postulante para examen de admisión2019. San Lorenzo, Paraguay

Facultad de Ciencias Químicas UNA (2017) Guía del postulante para examen de admisiónAño 2018. San Lorenzo, Paraguay

García Guadilla, C. (1996) Conocimiento, educación superior y sociedad en América Latina. Caracas: Centro de Estudios del Desarollo-CENDES

Garratte, L., Montenegro, J., Fava, M., \& Alcoba, J. (2018) Políticas de acceso a la universidad en Argentina. Estrategias institucionales y perspectivas en conflicto en la carrera de ingeniería. Revista Cubana de Educación Superior, 134-147

Ministerio de Industria y Comercio. (2014) MIC. Recuperado de http://www.mic.gov.py/mic/site/mic/univ1.php

Parra, O. (2012). Los sistemas de acceso, normativas de permanencia, y estrategias de tutoría y retención de estudiantes de educación superior en Paraguay. Asunción.

Pérez Lindo, A. (2009). ¿Para qué educamos hoy? Buenos Aires: Biblos.

Pesántez Avilés, F., Martín, E., \& Bojorque, R. (2015). Una mirada crítica al sistema de acceso a la Educación Superior ecuatoriano. Revista Cubana de Educación, 63-76.

Ponce, J., \& Carrasco, F. (2016). Acceso y equidad a la educación superior y posgrado en el Ecuador, un enfoque descriptivo. Revista Latinoamericana Y Acción Pública. FLACSO, Sede Ecuador, 9-22.

Rabossi, M. (2014). Acceso-Ingreso A Las Universidades Nacionales Argentinas: Permisividad Y Consecuencias. Páginas De Educación, 81-103.

Rivarola, D. M. (2003). La Educación Superior Universitaria en Paraguay. Asunción: Ediciones y Arte S.R.L.

Robledo, M. (2017). Gobernanza de la educación superior. El rol del aseguramiento de la calidad. Los casos de Argentina y Chile. Rev.cient.estud.investing. VI Foro del Investigador.

Universidad del Pacífico. (21 de Setiembre de 2018). Obtenido de https://www.upacifico.edu.py/Informes/InformesUP/categorias/218

Vergara Díaz, G., \& Peredo López, H. (2017). Relación del desempeño académico de estudiantes de primer año de universidad en Chile y los instrumentos de selección para su ingreso. Educación 